\title{
Stereodynamics of molecular photodissociation
}

\author{
J P Simons \\ Chemistry Department, The University, Nottingham NG7 2RD, UK
}

\begin{abstract}
The application of tunable, polarised pulsed laser techniques, in probing molecular photodissociation, is providing unparalleled insight into their three-dimensional, stereodynamics. A survey of some of the most recent developments is presented, illustrated by studies of hydrogen peroxide, t-butyl hydroperoxide, t-butyl nitrite and nitric acid.
\end{abstract}

\section{INTRODUCTION}

When the absorption of a photon by a polyatomic molecule so disturbs its electronic structure as to promote its fragmentation, it is not surprising that bond-breaking is accompanied by other structural changes. If the absorption spectrum associated with the electronic transition is a broad continuum, apart from reflecting a fleeting lifetime for the electronically excited molecule, and most likely a "half-collisional" direct dissociation patbway, the spectrum conveys no information on the dynamics of the dissociation process or in particular on its stereodynamics. It is these that convey the detailed, three-dimensional information relating to the changing geometries of the photoexcited molecule. The entry into this three-dimensional world is through the door of 'photofragment mapping', i.e. through probing both the scalar (energy) distributions and the vector (linear and angular momentum) correlations among the recoiling molecular (or atomic) fragments $[1]$.

Two alternative strategies have been developed to achieve this. The first involves various methods of photofragment imaging $[2,3,4]$; the most advanced systems employ laser ionisation and a multiple detection array to display the angular distribution of the ionised fragment trajectories [4], while time of flight measurements provide their velocity/momentum distributions and polarised laser beams provide the essential reference frame upon which the molecular image can be projected. The second strategy exploits the polarisation and in particular, the narrow line-width of tunable dye laser systems; the polarised laser induced fluorescence excitation, or multiple photon ionisation spectra of the molecular fragments are recorded at sub-Doppler resolution [5-7] in a laser pump (photolysis)-probe experiment. Analysis of the integrated intensity distributions in the resolved photofragment spectra provides their energy/quantum state distributions. Analysis of the Doppler contours of individual spectral lines reveals their velocity distributions. Finally, and most importantly, analysis of the Doppler contours under alternative photolysis - probe laser beam polarisation and propagation geometries reveals the vector correlations (average angles) between the molecular electronic transition dipole $\mu_{p}$ (referenced to the photolysis beam polarisation $\varepsilon_{p}$ ), the fragment recoil velocity $\mathbf{v}$ and rotational angular momentum $j$. [8].

In the last few years, these sophisticated new techniques have been used to reveal the detailed stereodynamics of a range of polyatomic molecules in quite unprecedented detail. This lecture is designed as an hors d'ouvre which will comprise a discussion of some exemplary direct photodissociation systems including $\mathrm{H}_{2} \mathrm{O}_{2},\left(\mathrm{CH}_{3}\right)_{3} \mathrm{COOH},\left(\mathrm{CH}_{3}\right)_{3} \mathrm{CONO}_{\text {and }} \mathrm{HNO}_{3}$.

\section{VECTOR CORRELATIONS}

The correlation between the photofragment recoil velocity $\mathbf{v}$ and the polarisation vector $\varepsilon_{p}$ of the photolysis beam (and thus the parent molecular transition dipole $\mu_{p}$ ) is expressed by the translational anisotropy, $\beta \square 2\left\langle\mathrm{P}_{2}\left(\boldsymbol{\mu}_{\mathrm{p}} \cdot \mathbf{v}\right)\right\rangle^{\dagger}$ the first member of an interlocking triangle of vector pair correlations. The other two, the rotational alignment,

$+\left\langle\mathrm{P}_{2}(\hat{\mathrm{a}} \cdot \hat{\mathrm{b}})\right\rangle=\frac{3\langle\hat{\mathrm{a}} \cdot \hat{\mathrm{b}})^{2}-1}{2}$ where $\hat{\mathrm{a}}$ and $\hat{\mathrm{b}}$ are unit vectors 
$A_{0}^{(2)}=\frac{4}{5}\left\langle P_{2}\left(\hat{\mu}_{p}, \hat{j}\right)\right\rangle$ and the $(\mathbf{v}, \mathbf{j})$ correlation, $\left\langle P_{2}(\hat{\mathbf{v}}, \hat{\mathbf{j}})\right\rangle$ both involve the rotational angular momentum of the recoiling fragments. The three correlations can take values lying between the limits

$$
\begin{aligned}
& 2 \geqslant \beta \geqslant-1 \\
& \frac{4}{5} \geqslant A_{0}^{(2)} \geqslant-\frac{2}{5} \\
& 1 \geqslant(\hat{\mathbf{v}}, \hat{\mathbf{j}}) \geqslant-\frac{1}{2}
\end{aligned}
$$

with positive and negative values representing the extremes of parallel or perpendigular alignment. The measured translational anisotropy, $B$ and the rotational alignment $A_{0}$, can both be 'degraded' by parent molecular rotation either because the dissociation time is long enough to allow significant rotation of the transition dipole $\mu_{\mathrm{p}}$ away from its initial alignment with respect to $\varepsilon_{p}$, or through the introduction of a tangential (orbital) velocity component. Their interpretation may also be complicated when there is an overlap of two (or more) distinct dissociation continua, with different symmetries, and hence different transition dipolar alignments.

The ( $\mathbf{v}, \mathbf{j}$ ) correlation does not involve the initial transition dipole $\mu_{p}$; it is an exit channel property, reflecting the dynamics at the instant of fragment separation. None the less, it too may be influenced by the parent molecular rotation, through angular momentum conservation. This may reduce or enhance the effect of vibrational angliar motion, e.g. torsion about the breaking bond, which tends to align $\mathrm{j} \| \mathbf{v}$ or perpendicular bending motions, which align $j \perp v$.

The pair-wise (and higher) correlations between $\varepsilon_{p}\left(\mu_{\rho}\right), \mathbf{v}$ and $\mathbf{j}$ are fundamental stereodynamical indicators: when they are strong they reflect strong steric influences on the recoiling fragments, from which it is possible to infer the details of the molecular changes which accompany photodissociation. Strong correlations exert a profound influence on both the Doppler profiles and the relative integrated intensities of individually resolved spectral features in the LIF excitation spectra of the recoiling fragments. This occurs because each of the photon absorption steps associated with the molecular photodissociation and the fragment LIF probing - are necessarily anisotropic processes. If the photodissociation dynamics generate strongly aligned (or with a circularly polarised photolysis beam, oriented) fragments, their absorption/fluorescence excitation spectra will be strongly polarization dependent [5-10]. The theoretical machinery required to extract the vectorial data from the experimental spectral contours has been developed by Zare [9], Dixon [8], Houston [6] and Docker [11] and will not be discussed here. Although. complex it is simple to apply in systems where the fragments recoil with a single, or at

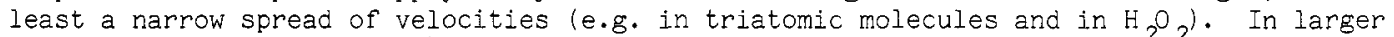
polyatomic molecular photodissociation systems such situations are more likely the exception than the rule, since a broad spread of internal energies in a polyatomic fragment necessarily leads to a corresponding spread of recoil kinetic energies/velocities (through total energy conservation) and a consequent blurring of the recorded Doppler profiles. In practice, however, this has not proved to be a severe problem; the development of Fourier Transform techniques $[12,13]$ for Doppler spectral inversion will further enhance the determination of final energy distributions and hence the precision of the derived vectorial data.

\section{A BENCH-MARK SYSTEM, $\mathrm{H}_{2} \mathrm{O}_{2}$}

In its ground electronic state, HOOH adopts an eclipsed equilibrium geometry, with a dihedral angle $120^{\circ}$ : a small torsional barrier in the trans-planar configuration leads to a splitting of the zero-point vibrational level. U.-v photon absorption is associated with two low-lying electronic transitions, populating the $\mathbb{A}^{1} \mathrm{~A}\left(4 \mathrm{~b}\left(\mathrm{n}_{0}\right) \rightarrow 5 \mathrm{~b}\left(\sigma_{0}^{*}-0\right)\right)$ and $\widetilde{B} \perp \mathrm{B}\left(5 \mathrm{a}\left(\mathrm{n}_{0}\right) \rightarrow 4 \mathrm{~b}\left(\sigma_{0}^{*}-0\right)\right)$ continua. The transitions, not surprisingly, are associated with broad continuum absorption peaking at $\sim 180 \mathrm{~nm}$, but extending a very broad and weak tail towards longer wavelengths. Direct photodissociation can even be effected with blue light (vide infra). Although the $\widetilde{A}$ and $\widetilde{B}$ continuum states are strongly repulsive along the $\mathrm{HO}-\mathrm{OH}$ coordinate, their preferred angular geometries are respectively trans $\left(\widetilde{A}^{1} A\right)$ and cis $\left(\widetilde{B}^{1} B\right)$ - planar. Polarised laser photodissociation at wavelengths between $248 \mathrm{~nm}$ and $308 \mathrm{~nm}$ [14-16] generates rapidly recoiling OH fragments which can be detected via LIF spectroscopy. A coaxial pump-probe geometry reveals characteristic 'forward-backward' Doppler spectral profiles, associated with recoil velocities $\mathbf{v}$ directly perpendicular to the transition dipole $\mu_{p}$. The translational anistropy, $\beta$, which lies close to the Iimiting value $\beta=-1$ (see $f i g .1$ ), identifies the principal component in the mid $u .-v$ continuum as $\left(\widetilde{A}^{1} A+\widetilde{X}^{1} A\right)$, with $\mu_{p}$ lying along the $C_{2}$ axis, perpendicular to the $0-0$ bond. At shorter and longer photolysis wavelengths however, the increasing contribution from a centrally peaked, 'sideways' Doppler component, reveals the overlapping $\widetilde{\mathrm{B}}^{1} \mathrm{~B} \leftarrow \widetilde{\mathrm{X}}^{1} \mathrm{~A}$ continuum for which the transition dipole $\mu_{p}$ lies near parallel to the $0-0$ axis [17]. At 
(a)

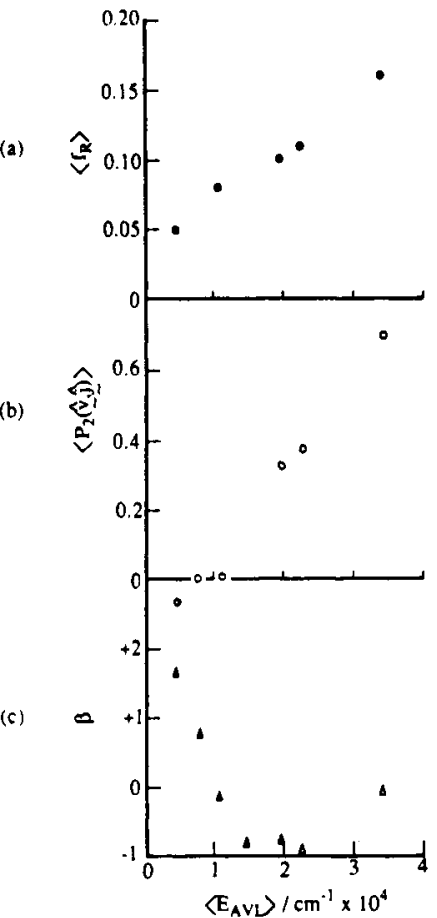

Fig. 1. Absorbed photon energy dependence of rotational energy disposal $\left\langle f_{R}\right\rangle$, the $(v, j)$ correlation and the translational anisotropy $B$, of $\mathrm{OH}$ fragments generated via photodissociation of $\mathrm{H}_{2} \mathrm{O}_{2}$.

the longest wavelengths, the translational anistropy changes sign and approaches the $\mu_{p} \| \mathbf{v}$ limiting value $\beta=+2$. The narrowing Doppler widths of the recoiling fragments also confirms a single photon photodissociation pathway, even when the energy of the (very weakly) absorbed photon is not too far above the dissociation threshold, see fig. 2 .
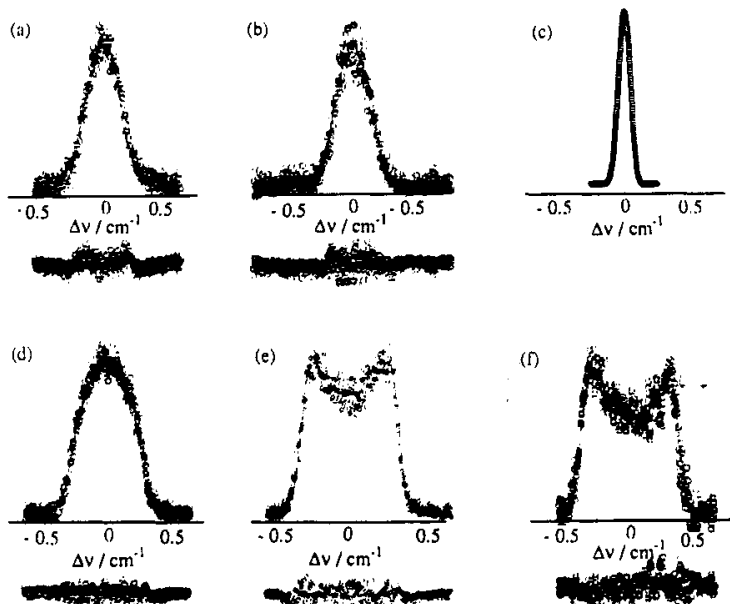

Fig. 2. Doppler resolved profiles of the $R_{2}(1)$ feature in the A-X L,IF spectra of OH fragments, generated via photodissociation of $\mathrm{H}_{2} \mathrm{O}_{2}$ and recorded using coaxial photolysis-probe beam geometry.
(a) $465 \mathrm{~nm}$ (b) $455 \mathrm{~nm}$ (d) $390 \mathrm{~nm}$
(e) $351 \mathrm{~nm}$ (f) $308 \mathrm{~nm}$;
(c) minimum experimental resolution.

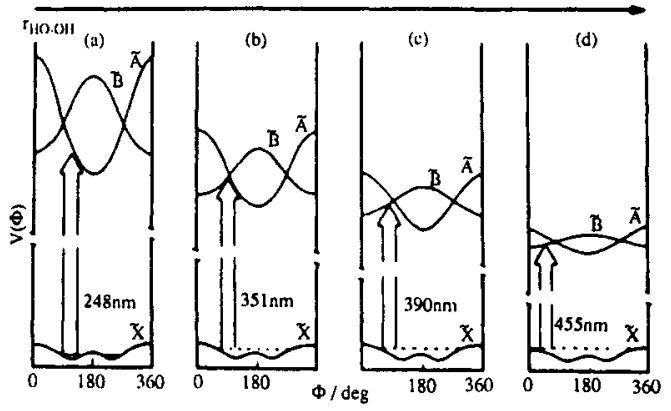

Fig. 3. Schematic sections through the $\widetilde{X}, \widetilde{A}$ and $\widetilde{B}$ state potential energy surface of $\mathrm{H}_{2} \mathrm{O}_{2}$. The vertical arrows indicate the probable shift in the mean torsional angle $\Phi$, of molecules absorbing radiation at wavelengths further and further to the red of the spectral maximum. (Based on data of R. Schinke and V. Staemmler, Chem. Phys.Lett., 145, 486 (1988)). 
The recoiling fragments carry no detectable energy in vibration, but at short wavelengths particularly, a significant fraction of the available energy, $E_{A V L} \simeq h v-D(H O-O H)$, is converted into rotation; this fraction $\left\langle f_{R}\right\rangle$ decreases almost linearly with decreasing $E_{A V L}$ (see fig. 1). At wavelengths far to the red of the vertical maximum, Franck-Condon considerations restrict photon absorption to the small sub-set of HOOH molecules with greatly extended internuclear $\mathrm{HO}-\mathrm{OH}$ separations. They will already be well on the way to the dissociation limit in the electronic continuum where the angle bending and torsional anisotropies will be greatly reduced (see fig. 3). The steady decline of 〈f $f_{R}$ with decreasing photon energy, implicates angle bending and torsion as the possible engines of fragment rotation. A strong torque associated with motion through a preferred planar geometry (trans via the $\widetilde{A}$ state or cis via the $\widetilde{B}$ state) would favour torsion and a preferred alignment of the fragment rotation $\mathbf{j} \| \mathbf{v}$, i.e. a 'propeller' motion. This expectation is confirmed by analysis of the Doppler resolved LIF profiles of the rotationally excited $O H$ fragments. Typical profiles, generated by photodissociation at $248 \mathrm{~nm}$, are shown in fig. 4; their high sensitivity to changes in the excitationdetection beam geometries or the polarisation of the related rotational feature ( $Q$-transition aligned parallel to $\mathrm{j} ; \mathrm{P}, \mathrm{R}$ - transition aligned perpendicular to $\mathrm{j}$ ) betrays a strong positive $(\mathbf{v}, \mathbf{j})$ correlation. Quantitative data over a range of photolysis wavelengths are summarised in fig. 1; the decreasing value of the correlation with decreasing energy confirms the decline of torsional anisotropy with increasing $\mathrm{HO}-\mathrm{OH}$ separation. A simple geometric construction, based on the average values for $(v \cdot j)^{2}$ and $\left(\mu_{p} j\right)^{2}$, determined at $248 \mathrm{~nm}[14]$ or $266 \mathrm{~nm}$ [16], indicates a trans-planar configuration as the preferred transition state geometry, again as expected for excitation into the $\widetilde{A}^{l} \mathrm{~A}$ continuum state.

\section{t-BUTYL HYDROPEROXIDE}

In t-butyl hydroperoxide, the introduction of the heavy t-butyl group should introduce two major changes

(a) The greatly increased mass of the tert-butoxy group will exert a powerful kinematic influence on the $\mathrm{OH}$ recoil trajectories.

(b) The many additional degrees of freedom will introduce a 'sponginess' in the skeletal framework and a considerable change in the partitioning of excess energy between internal and kinetic energy in the separating fragments.
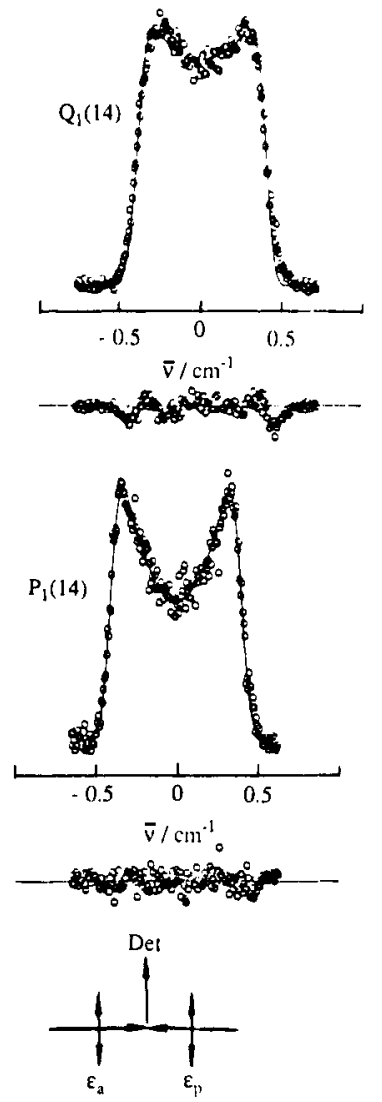
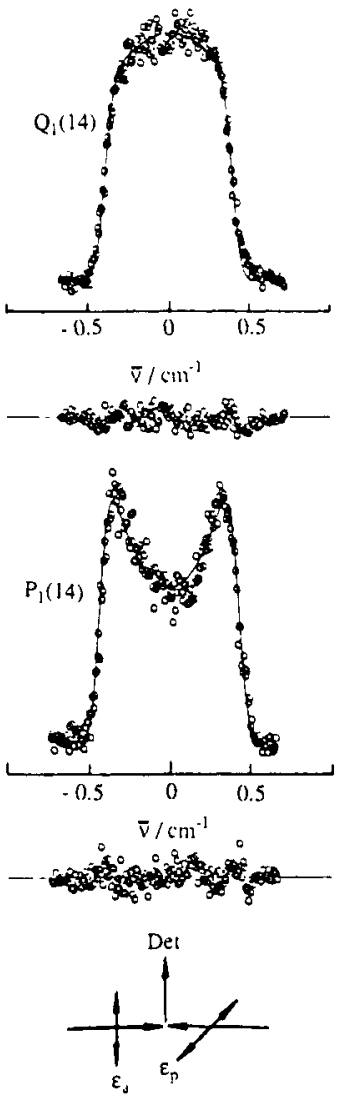

Fig. 4. Doppler resolved profiles of $\mathrm{P}_{1}(14)$ and $Q_{1}(14)$ features in the $A-X$ LIF spectra of $O H$ fragments, generated via photodissociation of $\mathrm{H}_{2} \mathrm{O}_{2}$ at $\overline{248} \mathrm{~nm}$, using alternative photolysisprobe beam geometries. 
Sub-Doppler probing of the LIF spectroscopy of the recoiling OH fragments has quantitatively confirmed both of these expectations [18].

The u.-v. continuum absorption of t-butyl hydroperoxide is little changed from that of $\mathrm{H}_{2} \mathrm{O}_{2}$ and the retention of its $\mathrm{n}_{\mathrm{O}} \rightarrow \sigma_{0}^{*} 0$ character can safely be assumed; thus photolysis in the mid u.-v. at $248 \mathrm{~nm}$, by analogy with $\mathrm{H}_{2} \mathrm{O}_{2}$, might be expected to generate $\mathrm{OH}$ fragments, recoiling with translational anisotropies, $B \sim-1$. Although still negative, the anisotropy is actually found to be much reduced, with an average value $B \simeq-0.3[18]$. (see table 1). The clue to understanding this change is found in the behaviour of the $(\mathrm{v}, \mathrm{j})$ correlation which is also greatly reduced relative to that in $\mathrm{H}_{2} \mathrm{O}_{2}$ and even changes sign in the lower rotational levels of the $\mathrm{OH}$ fragments. Although torsion about the $0-0$ bond still plays a role in promoting the fragment rotation, there is an increase in the relative contribution made by 'perpendicular' $\mathrm{OOH}$, angle bending torques. Their influence is also reflected in the increased rotational alignment $A_{0}^{(2)}$ (see table 1). An increase in the relative importance of bending as opposed to torsional torques, suggests an initial equilibrium geometry much closer to trans-planar - indeed 'ab initio' calculations for the related alkyl peroxide $\mathrm{CH}_{3} \mathrm{OOH}$, predict a much reduced torsional barrier about the $0-0$ bond [19]. Bending about the $\mathrm{OOH}$ angle transfers perpendicular rotational angular momentum to

TABLE 1 Mean vector correlations in $\mathrm{OH}$ fragments generated through photodissociation of t-butyl hydroperoxide at $248 \mathrm{~nm}$

\begin{tabular}{cccc}
$\begin{array}{c}\text { Rotational } \\
\text { level } \\
\mathrm{N}\end{array}$ & $\begin{array}{c}\text { Transational } \\
\text { anisotropy } \\
\beta\end{array}$ & $\begin{array}{c}\text { Rotational } \\
\text { alignment } \\
(2)\end{array}$ & $(\mathbf{v}, \mathbf{j})$ correlation \\
\hline 4 & -0.31 & +0.16 & -0.15 \\
10 & -0.27 & +0.14 & +0.15 \\
$10+$ & $\underline{-0.86}+$ & $+0.10+$ & $+0.35+$ \\
\hline
\end{tabular}

+ comparative data for $\mathrm{H}_{2} \mathrm{O}_{2}$ [14]

the OH fragment; on the other hand, because of the inertia of the heavy t-butyl group bending about the complementary $\left(\mathrm{CH}_{3}\right)_{3} \mathrm{COO}$ bond angle during dissociation will propel the recoiling $\mathrm{OH}$ fragment through a spiral trajectory. The resulting 'sling-shot' dynamics rotates the final recoil velocity $\mathbf{v}$ away from its initial perpendicular alignment with respect to the parent molecular transition dipole, $\mu_{p}$; hence $\beta \simeq-0.3$ rather than -1 .

The 'sponginess' of the t-butoxy group is revealed by the 'blurring' of the OH Doppler profiles and the reduced kinetic energy release. The reduction reflects the absorption of around one third of the available energy into ro-vibrational motion in the t-butoxy fragment, while the 'blurring' reflects the broad spread of internal energies around the mean value. The energy partitioning is in good agreement with the predictions of a simple impulsive model, consistent with the assumed direct dissociation mechanics. The average internal excitation in the t-butoxy fragments is more than sufficient to promote their unimolecular decomposition

$$
\left(\mathrm{CH}_{3}\right)_{3} \mathrm{CO} \rightarrow \mathrm{CH}_{3}+\left(\mathrm{CH}_{3}\right)_{2} \mathrm{CO}, \Delta \mathrm{E}_{0} \simeq+50 \mathrm{~kJ} \mathrm{~mol}^{-1}
$$

\section{t-BUTYL NITRITE}

The equilibrium geometry of t-butyl nitrite is predicted to be trans-planar [20]. Its u.-v absorption has been assigned to two distinct $n_{0} \rightarrow \pi^{*}$ electronic transitions: the first, $\widetilde{A}^{1} A^{\prime \prime} \leftarrow \widetilde{X}^{1} A$ ' originating on the terminal $O$ atom is associated with a diffuse banded spectrum, while the second, $\widetilde{\mathrm{B}}^{1} A^{\prime}+\widetilde{\mathrm{X}}^{I_{A}}$ originates on the central 0 atom, and generates a structureless continuum. It is this continuum which is accessed by photon absorption at $248 \mathrm{~nm}$. If the trans-planar symmetry is retained during the photodissociation

$$
\left(\mathrm{CH}_{3}\right)_{3} \mathrm{CONO}+\mathrm{hv}(248 \mathrm{~nm}) \rightarrow\left(\mathrm{CH}_{3}\right)_{3} \mathrm{CO}+\mathrm{NO}\left(\mathrm{X}^{2} \Pi\right)
$$

which follows photon absorption, the following stereodynamical features might be anticipated: -

(a) alignment of the molecular transition dipole $\mu_{p}$ in the molecular plane

(b) strong rotation of the NO fragment promoted by an impulsive transfer into angular momentum about its centre of mass, and hence directed perpendicular to $\mu_{p}$.

(c) a consequent rotational alignment $A_{0}^{(2)} \rightarrow-0.4$, and $(v, j)$ correlation $\rightarrow-\mu_{0} .5$.

(d) a translational anisotropy $\beta$ anywhere between the two extremes $2 \geqslant \beta \geqslant-1$, dependent on the alignment of $\mu_{p}$ relative to the RO-NO bond. 
TABLE 2 Mean vector correlations in (NO), "fragments" generated through photodissociation of t-butyl nitrite in the $\widetilde{\mathrm{B}}^{1} \mathrm{~A}^{\prime}+\widetilde{\mathrm{X}}^{1} \mathrm{~A}^{\prime}$ continuum at $248 \mathrm{~nm}$

\begin{tabular}{cccc}
$\begin{array}{c}\text { Rotational } \\
\text { level } \\
\mathrm{N}\end{array}$ & $\begin{array}{c}\text { Translational } \\
\text { anisotropy } \\
\beta\end{array}$ & $\begin{array}{c}\text { Rotational } \\
\text { alignment } \\
\mathrm{A}_{0}^{(2)}\end{array}$ & $(\mathbf{v}, \mathbf{j})$ correlation \\
\hline 51 & +1.06 & -0.30 & -0.51 \\
\hline
\end{tabular}

Table 2 shows the experimental values for each of the vector correlations determined for rotationally excited NO "fragments" in levels $j=40-60$ and carrying one quantum of vibrational excitation [18]. As expected, the recoiling No is almost perfectly aligned perpendicular to the molecular plane and to the recoil velocity: no significant dynamical contribution from out-of-plane torsional motion is apparent. The translational anisotropy is strongly positive and $\mu_{p}$ must be aligned close to the axis of the central 0-NO bond.

As with t-butyl hydroperoxide, the pattern of energy disposal lies close to an 'impulsive' expectation, and once again the t-butoxy group absorbs a high fraction of the available energy - this time approaching 50\% - and more than sufficient to promote the unimolecular decomposition (1).

\section{$\mathrm{HONO}_{2}$}

Nitric acid is a planar molecule in its ground electronic state. Its u.-v absorption spectrum is entirely continuous displaying a weak, broad maximum centred around $270 \mathrm{~nm}$ and a much stronger peak at $\sim 185 \mathrm{~nm}$. Ab initio calculations [21] predict three successive low-lying electronic transitions populating two states of A" symmetry, populated via $\mathrm{n}_{2} \rightarrow: \pi^{*}$ transitions. All three excited states are predicted to be non-planar. Photon absorption at any wavelength in the continuum leads to the dissociation(s)

$$
\mathrm{HONO}_{2}+\mathrm{h \nu} \rightarrow \mathrm{OH}\left(\mathrm{X}^{2} \Pi\right)+\mathrm{NO}_{2}\left(\widetilde{\mathrm{X}}^{2} \mathrm{~A}_{1}, \widetilde{\mathrm{A}}^{2} \mathrm{~B}_{2}\right)
$$

Doppler resolved LIF spectral profiles of the recoiling, rotationally excited OH fragments, generated at $280 \mathrm{~nm}$ near the long wavelength peak, and recorded using two alternative excitation-detection geometries, are shown in fig. 5 [22]. The appearance of the 'tell-tale', forward-backward Doppler shape for the perpendicular (fig. 5 (a)), rather than the co-axial geometry, reflects a tendency for recoil in directions parallel to the molecular transition dipole $\mu_{p}$ and suggests that $\mu_{p}$ lies closely parallel to the $\mathrm{N}-\mathrm{OH}$ bond.

Detailed analysis of the Doppler profiles gives a value for the translational anisotropy, $\beta \approx+0.8$ however, less than the limiting value $\beta=+2$. A spiral trajectory, resulting from a planar $\rightarrow$ pyramidal geometry change in the excited state, and a mean out-of-plane angle of $40^{\circ}$, readily accommodates the difference. Ab initio calculation predicts an out-of-plane angle of $31^{\circ}$ [21]. If the stereodynamics had remained coplanar and in the absence of any torsional torque about the breaking $\mathrm{N}-\mathrm{OH}$ bond, the fragment rotational angular momentum $j$ would have been directed perpendicular both to $\mu_{p}$ and to the recoil velocity $\mathbf{v}$, and the rotational alignment and $(\mathbf{v}, \mathbf{j})$ correlations would both have been negative. In practice, both are positive (see table 3 )! There is a broad tendency for $\mu_{p}\|v\| j$.

(a)

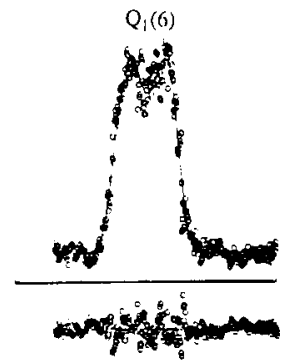

(b)

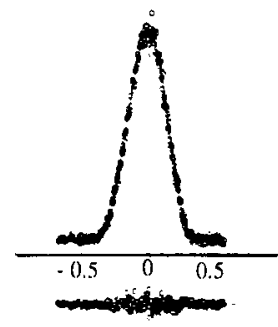

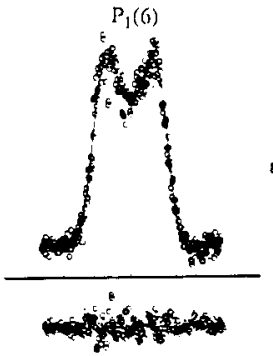

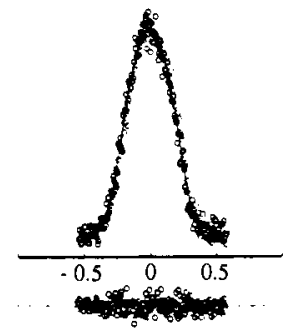

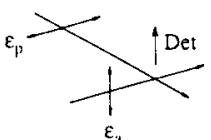

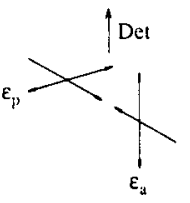

Fig. 5. Doppler resolved profiles $P_{1}(N), Q_{1}(N)$ features in the $A-X$ LIF spectra of $\mathrm{OH}$ fragments, generated via photodissociation of $\mathrm{HNO}_{3}$ at $280 \mathrm{~nm}$, using alternative photolysis-probe beam geometries. 
TABLE 3 Mean vector correlations in $(\mathrm{OH})_{\mathrm{v}=0}$ fragments generated through photodissociation of HNO at $280 \mathrm{~nm}$ [22] and $225 \mathrm{~nm}$ [23]

\begin{tabular}{ccccc}
$\begin{array}{c}\text { Rotational } \\
\text { level } \\
\mathrm{N}\end{array}$ & $\begin{array}{c}\text { Translational } \\
\text { anisotropy } \\
B\end{array}$ & $\begin{array}{c}\text { Rotational } \\
\text { alignment } \\
(2)\end{array}$ & $(\mathbf{v}, \mathbf{j})$ correlation \\
\hline $280 \mathrm{~nm}$ & 6 & $\begin{array}{l}+0.88 \\
+0.8\end{array}$ & $\begin{array}{l}+0.14 \\
+0.17\end{array}$ & $\begin{array}{l}+0.29 \\
+0.52\end{array}$ \\
\hline $225 \mathrm{~nm}$ & 10 & +0.17 & +0.08 & +0.24 \\
\hline
\end{tabular}

The detailed values of the three stereodynamical indicators can be understood if

(a) the $\mathrm{ONO}_{2}$ framework of the photoexcited $\mathrm{HONO}_{2}$ becomes pyramidal, and the terminal $0-\mathrm{H}$ group becomes twisted out of the initial plane, and

(b) the excited state has A' symmetry.

'Ab initio' calculations [21] (conducted after the experiments!) confirm (a) but predict A" electronic symmetry for the excited molecule. However, the transition is extremely weak, with an oscillator strength, $f \simeq 2 \times 10^{-6}$; a vibronic transition involving out-of-plane deformation reconciles experiment with theory.

Stereodynamical studies have also been made for $\mathrm{HONO}_{2}$ molecules excited at $225 \mathrm{~nm}$ [23], close to the predicted wavelength for the vertical transition $\widetilde{B}^{1} A^{\prime \prime}+\widetilde{X}^{1} A^{\prime}[21]$. The predicted polarisation of the transition dipole perpendicular to the initial molecular plane, favours a negative value for the translational anisotropy, $\beta$, but once again the generation of a spiral recoil trajectory following the planar $\rightarrow$ pyramidal geometry change reduces $\beta$ from its limiting value: indeed it actually changes sign, to give $\beta \simeq+0.2$, corresponding once again, to a mean out-of-plane angle of $40^{\circ}$, cf. the predicted deformation through $31^{\circ}$ [21]. Finally the (modestly) positive value found for the $(\mathbf{v}, \mathbf{j}$ ) correlation, reveals the (predicted) torsion about the $\mathrm{N}-\mathrm{OH}$ bond.

The partitioning of energy beween kinetic and internal motions is profoundly influenced by the strong antibonding character of the $\pi * \mathrm{~m} .0$. The equilibrium $\mathbb{N}-0$ bond lengths in the $\mathrm{NO}_{2}$ group are predicted to increase by $80-100 \mathrm{pm}$ in the two $\mathrm{n} \rightarrow \pi^{*}$ states. Since the bond lengths in free $\mathrm{NO}_{2}$, are virtually the same as those in the ground state of $\mathrm{HONO}_{2}$, simple Franck-Condon considerations predict high vibrational excitation in the $\mathrm{NO}_{2}$ product. Not surprisingly, detailed analysis of the Doppler profiles, establishes the concentration of around two thirds of the available energy into internal excitation of the $\mathrm{NO}_{2}$, both at $280 \mathrm{~nm}\left(\widetilde{\mathrm{A}}^{\mathrm{l}} \mathrm{A}^{\prime \prime}\right)$ and $225 \mathrm{~nm}\left(\widetilde{\mathrm{B}}^{\mathrm{l}} \mathrm{A}^{\prime \prime}\right)[22,23]$. Fluorescence from electronically excited $\mathrm{NO}_{2}$ has also been detected following photodissociation at $241 \mathrm{~nm}$ [24].

\section{CONCLUSION}

The use of polarised, Doppler resolved laser probing of molecular photodissociation products to expose both their scalar and vectorial properties, has now become a wellestablished stereo-dynamic strategy. New developments include the measurement of rotational alignment of dissociating systems in real time, using ultra-fast pulsed laser systems [25]; the probing of oriented (unidirectional) systems [26] and oriented fragments [27]; the development of Fourier Transform inversion methods for Doppler profile analysis $[12,13]$ to better cope with the common situation of broadly distributed energy disposals; and the use of three-dimensional photofragment mapping strategies to probe intramolecular vibrational/rotational energy redistribution [28].

\section{Acknowledgements}

The author is very greatly indebted to his colleagues, past and present, who have allowed him to review their contributions: in particular Drs. A Hodgson, M Brouard, M T Martinez, $J$ August, M P Docker, and Messrs. C J Milne, J O'Mahony and J.-X Wang. Grants from SERC are also hereby acknowledged. 


\section{REFERENCES}

1. Molecular Photodissociation Dynamics", Adv. in Gas Phase Photochemistry and Kinetics, Ed. M.N.R. Ashfold and J.E. Baggott, Roy.Soc.Chem., 1987.

2. K.R. Wilson in "Excited State Chemistry", ed. J.N. Pitts, Gordon and Breach, New York, 1970.

3. A.M. Wodtke and Y.T. Lee, ref. (1), p.31

4. D.W. Chandler and P.L. Houston, J.Chem. Phys. 87, 1445 (1987).

5. J.P. Simons, J.Phys.Chem. 91, 5378 (1987).

6. P.L. Houston, J.Phys.Chem. 91,5388 (1987).

7. G.E. Hall and P.L. Houston, Ann.Rev.Phys.Chem. 40, 375 (1989).

8. R.N. Dixon, J.Chem.Phys. $85,1866(1986)$.

9. A.C. Kummel, G.0. Sitz and R.N. Zare, J.Chem.Phys. 88, 7357 (1988).

10. D.A. Case, G R McClelland and D.R. Herschbach, Mol. Phys. 35, 341 (1978).

11. M.P. Docker, Chem.Phys. 135, 405 (1989); Chem. Phys. 125, 185 (1988).

12. A. Ticktin and J.R. Huber, Chem.Phys.Lett. $\overline{156,372}(1 \overline{989})$.

13. P. Enriques, R. Sayos and J.P. Simons, unpublished work.

14. (a) M.P. Docker, A. Hodgson and J.P. Simons, Faraday Discussions Chem. Soc. 82,25 $(1986)$.

(b) J. August, M. Brouard, M.P. Docker, A. Hodgson, C.J. Milne and J.P. Simons, Ber.Bunsenges. Phys.Chem. 92, 264 (1988).

15. K.-H. Gericke, S. Klee, F.J. Comes and R.N. Dixon, J.Chem.Phys. 85, 4463 (1986)

16. M. Brouard, M.T. Martinez, C.J. Milne, J.P. Simons and J.-X. Wang, Chem. Phys.Lett. 165,423 (1990).

17. C. Chevaldonnet, H. Cardy and A. Dargelos, Chem. Phys. 102, 55 (1986)

18. J. August, M. Brouard, M.P. Docker, C.J. Milne, J.P. Simons, R. Lavi, S. Rosenwaks and D. Schwartz-Lavi, J.Phys.Chem. 92,5485 (1988).

19. R.A. Bair and W.A. Goddard III, J.Amer.Chem.Soc. 104, 2719 (1982).

20. P. Tarte, J.Chem. Phys. 20, 1570 (1952).

21. Y.Y. Bai and G.A. Segal, J.Chem.Phys. in press.

22. J. August, M. Brouard and J.P. Simons, J.Chem.Soc.Faraday 2 84, 587 (1988).

23. M. Brouard, M.T. Martinez and J.P. Simons, unpublished work.

24. A. Sinha, R.L. Vander Wal and F.F. Crim, J.Chem.Phys. 91, 2929 (1989).

25. A.H. Zewail, J.Chem.Soc.Faraday 285,1221 (1989).

26. R.B. Bernstein, S.E. Choi and S. Stolte, J.Chem.Soc.Faraday 2 85, 1097 (1989)

27. E. Hasselbrink, J.R. Waldeck and R.N. Zare, Chem.Phys. 125, 191 (1988); J.Chem.Soc.Faraday 285,1044 (1989).

28. M. Brouard, M.T. Martinez, J. O'Mahony and J.P. Simons, J.Chem.Soc.Faraday 2 85, 1207 (1989); Mol.Phys. 69, 65 (1990); Phil.Trans.Roy.Soc. in press, (1990). 